\title{
Complexes Whose Boundaries Cannot Be Pushed Around
}

\author{
Ethan D. Bloch* \\ Bard College, Annandale-on-Hudson, NY 12504, USA
}

\begin{abstract}
Let $K^{n} \subset \mathbb{R}^{n}$ be a triangulated $n$-ball. Examples are given to show that unlike in the two-dimensional case, the following hold for all $n \geq 3$ : (1) there are nonconvex $K^{n}$ with no convex simplexwise linear embeddings $K^{n} \rightarrow \mathbb{R}^{n}$, even though there are strictly convex simplexwise linear embeddings $\partial K^{n} \rightarrow \mathbb{R}^{n} ;(2)$ there are convex $K^{n}$, with no spanning simplices, such that not every simplexwise linear embedding $f: \partial K^{n} \rightarrow \mathbb{R}^{n}$ with convex image can be extended to a simplexwise linear embedding of $K^{n}$; (3) there are convex $K^{n}$ such that the space of simplexwise linear homeomorphisms of $K^{n}$, fixed on $\partial K^{n}$, is not path connected.
\end{abstract}

\section{Introduction}

Let $K^{n} \subset \mathbb{R}^{m}$ be a simplicial complex; we use $K^{n}$ to denote both the simplicial complex and its underlying topological space. A map $f: K^{n} \rightarrow \mathbb{R}^{p}$ is called simplexwise linear (abbreviated SL) if $f \mid \sigma$ is an affine linear map for every simplex $\sigma$ in $K^{n}$. Note that an SL map is determined by what it does to the vertices. From now on assume that $K^{n}$ is an $n$-ball in $\mathbb{R}^{n}$. Let $L\left(K^{n}\right)=\{S L$ homeomorphisms $K^{n} \rightarrow K^{n}$ fixing $\partial K^{n}$ pointwise $\}$. (We can also think of $L\left(K^{n}\right)$ as the space of all triangulations of an $n$-ball, with given boundary, that are simplicially isomorphic to $K^{n}$.) If $n=2$, a number of results are known about $L\left(K^{2}\right)$ and various spaces of SL embeddings and near-embeddings of $K^{2}$; see [1]-[6]. In dimensions three and higher very little is known. One of the few positive results is in [10], where it is proved that, for any $n \geq 2$, if $K^{n}$ has at most two interior vertices, then $L\left(K^{n}\right)$ is contractible (even if $K^{n}$ is not convex).

On the other hand, it is known that some two-dimensional results do not hold in three dimensions. For example, it is proved in [6] that if $K^{2}$ is convex, then $L\left(K^{2}\right)$ is homeomorphic to some Euclidean space; in particular, it is contractible.

\footnotetext{
* Partially supported by NSF Contract DMS -8503388 .
} 
This result is shown to be false in three dimensions in [13], where the following theorem is proved.

Theorem 1 (Starbird). For any 3-ball $K^{3} \subset \mathbb{R}^{3}$, there is a subdivision $K^{\prime}$ of $K^{3}$ such that $L\left(K^{\prime}\right)$ is not connected.

In particular, $K^{3}$ in the above theorem can be chosen to be strictly convex ( $K^{n}$ is strictly convex if it is convex, and for every simplex $\sigma$ of $\partial K^{n}$, there is an $(n-1)$-plane $N$ of $\mathbb{R}^{n}$ such that $N \cap K^{n}=\sigma$.) In Example 3 of this paper we show that such examples exist in all dimensions greater than two by proving the following theorem. (In our example strict convexity is replaced by convexity; it would be of interest to know if there are strictly convex examples.)

Theorem 2. For all $n \geq 3$, there is a convex $n$-ball $K^{n}$ such that $L\left(K^{n}\right)$ is not connected.

This result essentially answers Question 6 of [8]. For $n=3$ our example is somewhat different from Starbird's: our example uses linking, whereas Starbird's uses knots. Our result in three dimensions is not as strong as Starbird's.

A much more elementary result which holds in two dimensions but fails in higher dimensions is the following theorem. Some terminology: we say that an embedding of $\partial K^{n}$ is starlike, convex, or strictly convex if it bounds a starlike, convex, or strictly convex $n$-ball; a simplex in $K^{n}$ which has all its vertices in $\partial K^{n}$, but is not itself in $\partial K^{n}$, is called a spanning simplex.

Theorem 3 (Bing and Starbird). Let $K^{2} \subset \mathbb{R}^{2}$ be any 2-disk (not necessarily convex, starlike, etc.), and let $f: \partial K^{2} \rightarrow \mathbb{R}^{2}$ be an $S L$ embedding. Then either of the following two conditions implies that $f$ can be extended to an $S L$ embedding $K^{2} \rightarrow \mathbb{R}^{2}$ :

(i) $f\left(\partial K^{2}\right)$ is starlike, and $K^{2}$ has no spanning 1 -simplices.

(ii) $f\left(\partial K^{2}\right)$ is strictly convex.

This is Theorems 2.1 and 2.2 of [1]. Note that part (ii) of the theorem is false if "strictly convex" is replaced by "convex". That this theorem fails in dimension three can be seen from the main example in [7], where a convex 3 -ball in $\mathbb{R}^{3}$ is given which cannot be SL re-embedded in $\mathbb{R}^{3}$ as a strictly convex ball; a theorem of Steinitz, however, implies that the boundary of any triangulated 3-ball has a strictly convex SL embedding in $\mathbb{R}^{3}$ (see Chapter 13 of [9]). In dimensions higher than three the examples of [7], as well as those of [12] (which have $n+4$ vertices in dimension $n$ ), do not show that the analog of Theorem 3 is false, since in these examples the boundary has no strictly convex SL embedding. However, in Example 1 we settle the higher-dimensional case by proving:

Theorem 4. For all $n \geq 3$, there is an $n$-ball $K^{n} \subset \mathbb{R}^{n}$ with $n+4$ vertices such that $K^{n}$ has no convex $S L$ embedding in $\mathbb{R}^{n}$, even though $\partial K^{n}$ has a strictly convex $S L$ embedding in $\mathbb{R}^{n}$. 
Note that our examples in the above theorem have the same number of vertices as in [12] (which is only for dimensions four and above); in dimension three our example has fewer vertices than in [7]. Since $n+4$ is minimal for what is proved in [12], we ask:

Question 1. Is $n+4$ the minimal number of vertices for the examples in Theorem 4 ?

The above theorem shows that the higher-dimensional analog of Theorem 3 is false by starting with nonconvex $\boldsymbol{n}$-balls; it might be conjectured that the analog holds if we start with convex $n$-balls. However, we prove:

\section{Theorem 5.}

(i) For all $n \geq 3$, there are strictly convex $n$-balls $K^{n} \subset \mathbb{R}^{n}$ with $n+3$ vertices such that not every strictly convex $S L$ embedding $f: \partial K^{n} \rightarrow \mathbb{R}^{n}$ can be extended to an $S L$ embedding of $K^{n}$. Moreover, $n+3$ is the minimal number of vertices for such an example.

(ii) For all $n \geq 3$, there are convex $n$-balls $K^{n} \subset \mathbb{R}^{n}$ with no spanning simplices such that not every convex $S L$ embedding $f: \partial K^{n} \rightarrow \mathbb{R}^{n}$ can be extended to an $S L$ embedding of $K^{n}$.

Part (i) of Theorem 5 is Example 2, and part (ii) is in Example 3.

\section{Example 1}

Some preliminaries. An $i$-plane $\Pi^{i}$ and a $j$-plane $\Pi^{j}$ in $\mathbb{R}^{i+j+1}$ are called skew if (1) $\Pi^{i} \cap \Pi^{j}=\varnothing$ and (2) the $i$ - and $j$-dimensional subspaces of $\mathbb{R}^{i+j+1}$ parallel to $\Pi^{i}$ and $\Pi^{j}$ intersect only at the origin. An $i$-simplex and a $j$-simplex in $\mathbb{R}^{i+j+1}$ are called skew if their affine spans are skew.

Let $\sigma^{i}, \tau^{j} \subset \mathbb{R}^{i+j+1}$ be skew $i$ - and $j$-simplices, respectively, and let $\sigma^{*} \in \sigma^{i}$ and $\tau^{*} \in \tau^{j}$ be arbitrary interior points. Let $A_{\sigma}, A_{\tau} \in\left\langle\sigma^{*}, \tau^{*}\right\rangle$ be any two distinct points such that $\boldsymbol{A}_{\sigma}$ is closer to $\tau^{*}$, and $\boldsymbol{A}_{\tau}$ is closer to $\sigma^{*}$. It is easy to verify that the $i$-sphere $S_{\sigma}=\left(A_{\sigma} * \partial \sigma\right) \cup \sigma$ and $j$-sphere $S_{\tau}=\left(A_{\tau} * \partial \tau\right) \cup \tau$ are disjoint, and are linked, in $\mathbb{R}^{i+j+1}$ (where * denotes join). We call $S_{\sigma}$ and $S_{\tau}$ coned links.

We now describe the examples of Theorem 4. Let $n \geq 3$ be given, let the following be points in $\mathbb{R}^{n}: E_{1}=(1,0, \ldots, 0), E_{2}=(0,1,0, \ldots, 0), \ldots, E_{n-1}=$ $(0, \ldots, 0,1,0), A=(-1,-1, \ldots,-1,0), U=(0, \ldots, 0,1)$, and $D=(0, \ldots, 0,-1)$. The following three facts can be verified straightforwardly using linear algebra:

(1) The convex hull of $\left\{E_{1}, \ldots, E_{n-1}, A, U, D\right\}$ has all $n+2$ of these points as vertices.

(2) The 1-simplex $\sigma=\left\langle E_{1}, U\right\rangle$ is skew to the $(n-2)$-simplex $\tau=$ $\left\langle E_{2}, \ldots, E_{n-1}, D\right\rangle$.

(3) The affine span of $\left\langle E_{3}, \ldots, E_{n-1}, A, U, D\right\rangle$ is an $(n-1)$-plane which separates $\mathbb{R}^{n}$ into two open half-spaces, with $E_{1}$ in one half-space and $E_{2}$ in the other. 
We construct $\partial K^{n}$. Take the boundary of $H=$ convex hull of $\left\{E_{1}, \ldots, E_{n-1}, A, U, D\right\}$ with its natural triangulation, and add two vertices $P$ and $Q$ as follows. Let $P$ be the barycenter of the $(n-1)$-simplex $\left\langle E_{1}, E_{3}, \ldots, E_{n-1}, A, D\right\rangle$, and let $Q$ be the barycenter of the $(n-1)$-simplex $\left\langle E_{2}, E_{3}, \ldots, E_{n-1}, A, U\right\rangle$. Triangulate $\partial K^{n}$ by taking the natural triangulation of $\partial H$, and starring on $P$ and $Q$. Note that by pushing $P$ and $Q$ out very slightly, $\partial K^{n}$ is seen to have a strictly convex embedding in $\mathbb{R}^{n}$. (In general we cannot budge a convex triangulation to get a strictly convex one, as seen in [7]; however, our $\partial K^{n}$ is so simple that we can do such a budge.)

We now construct the triangulation of $K^{n}$. First, we need to re-embed $\partial K^{n}$. Let the vertices $E_{1}, \ldots, E_{n-1}, A, U, D$ be as above. Let $\beta$ be a line segment from an interior point of $\sigma$ to an interior point of $\tau$ (where $\sigma$ and $\tau$ are defined in fact (2) above). Choose $P^{\prime}$ and $Q^{\prime}$ on $\beta$ so that $P^{\prime}$ is very close to $\sigma$ and $Q^{\prime}$ is very close to $\tau$. It is not hard to see that fact (3) above implies that, with vertices $E_{1}, \ldots, E_{n-1}, A, U, D, P^{\prime}$, and $Q^{\prime}, \partial K^{n}$ is still embedded. Consider $\partial K^{n} \cup$ $\left(P^{\prime} * \partial \tau\right) \cup\left(Q^{\prime} * \partial \sigma\right)$. By choice of $P^{\prime}$ and $Q^{\prime}$, the coned links construction above implies that the 1-sphere $\left(Q^{\prime} * \partial \sigma\right) \cup \sigma$ is disjoint from, and links, the $(n-2)$ sphere $\left(P^{\prime} * \partial \tau\right) \cup \tau$. Unfortunately, $\partial K^{n} \cup\left(P^{\prime} * \partial \tau\right) \cup\left(Q^{\prime} * \partial \sigma\right)$ is not embedded, the problem being that $Q^{\prime} * \partial \sigma$ intersects the $(n-1)$-simplex $\left\langle E_{1}, E_{3}, \ldots, E_{n-1}, D, P^{\prime}\right\rangle$ of $\partial K^{n}$, and $P^{\prime} * \partial \tau$ intersects the $(n-1)$-simplex $\left\langle E_{2}, E_{3}, \ldots, E_{n-1}, U, Q^{\prime}\right\rangle$ of $\partial K^{n}$. However, by budging $P^{\prime}$ off $Q^{\prime} * \partial \sigma$ (but staying in the affine span of $P^{\prime} * \partial \tau$ ), and then budging $Q^{\prime}$ off $P^{\prime} * \partial \tau$ (but staying in the affine span of $\left.Q^{\prime} * \partial \sigma\right)$, we can make $\partial K^{n} \cup\left(P^{\prime} * \partial \tau\right) \cup\left(Q^{\prime} * \partial \sigma\right)$ embedded, while

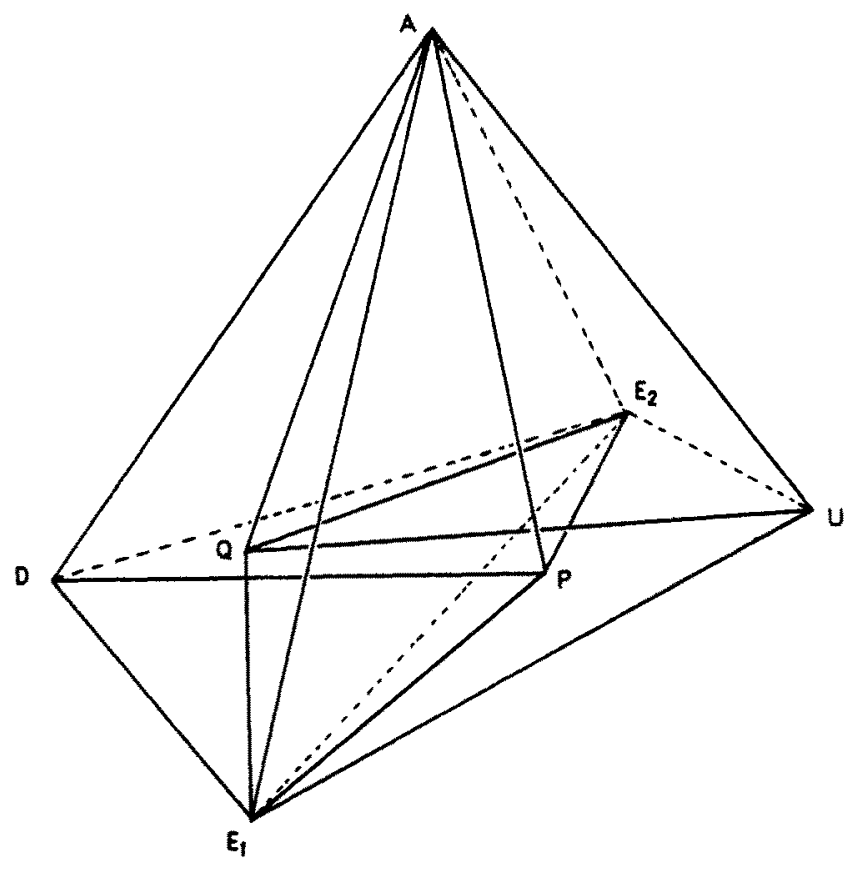

Fig. 1 
keeping $\left(Q^{\prime} * \partial \sigma\right) \cup \sigma$ disjoint from, and linked with, $\left(P^{\prime} * \partial \tau\right) \cup \tau$. Finally, we obtain a triangulation of $K^{n}$ by extending $\partial K^{n} \cup\left(P^{\prime} * \partial \tau\right) \cup\left(Q^{\prime} * \partial \sigma\right)$ to a triangulation of the bounded component of $\mathbb{R}^{n}-\partial K^{n}$, which can be done without subdividing $\partial K^{n} \cup\left(P^{\prime} * \partial \tau\right) \cup\left(Q^{\prime} * \partial \sigma\right)$, and without adding any new vertices. Note that the given embedding of $K^{n}$ is not convex, and has exactly $n+4$ vertices.

To complete this example, it remains to show that there is no convex embedding of $K^{n}$ in $\mathbb{R}^{n}$; assume otherwise, i.e., $K^{n}$ has been re-embedded as a convex $n$-ball. Consider the affine span $I I$ of $\left\langle E_{1}, \ldots, E_{n-1}, A\right\rangle$, an $(n-1)$-plane. Then $\Pi \cap \partial K^{n}=$ $\left(A * \partial\left\langle E_{1}, \ldots, E_{n-1}\right\rangle\right) \cup\left\langle E_{1}, \ldots, E_{n-1}\right\rangle$, which is an $(n-2)$-sphere, and which separates $\partial K^{n}$. Observe that $U$ and $Q$ are in one component of $\partial K^{n}-\left(\Pi \cap \partial K^{n}\right)$, whereas $D$ and $P$ are in the other component. $\Pi$ separates $K^{n}$ into two closed convex pieces, which only intersect in their boundaries; it is seen that $E_{1}, U, Q$ are all in one piece, and $E_{2}, \ldots, E_{n-1}, D, P$ are all in the other. It follows that $\left(Q^{\prime} * \partial \sigma\right) \cup \sigma$ is contained in one of these pieces, and $\left(P^{\prime} * \partial \tau\right) \cup \tau$ is contained in the other. This last fact implies that $\left(Q^{\prime} * \partial \sigma\right) \cup \sigma$ and $\left(P^{\prime} * \partial \tau\right) \cup \tau$ could not possibly be linked, contradicting the fact that these two spheres were linked in the original triangulation of $K^{n}$, and that linking is invariant under homeomorphism. Thus $K^{n}$ could not have been embedded as a convex ball.

Figure 1 shows the example when $n=3$; the complex has been rotated in $\mathbb{R}^{3}$ for ease of drawing.

\section{Example 2}

We construct the example of Theorem $5(i)$. Consider $\mathbb{R}^{n-2}=\mathbb{R}^{n-2} \times\{0\} \subset \mathbb{R}^{n-1}=$ $\mathbb{R}^{n-1} \times\{0\} \subset \mathbb{R}^{n}$. Let $\sigma^{n-2}$ be an $(n-2)$-simplex in $\mathbb{R}^{n-2}$ with barycenter at the origin. Let $L, R \in \mathbb{R}^{n-1}$ be $L=(0, \ldots, 0,-1)$ and $R=(0, \ldots, 0,1)$, and let $M^{n-1} \subset$ $\mathbb{R}^{n-1}$ be the simplicial complex $M^{n-1}=\left(L * \partial \sigma^{n-2}\right) \cup\left(R * \sigma^{n-2}\right)$ (where $*$ denotes join). Let $S, N \in \mathbb{R}^{n}$ be $S=(0, \ldots, 0,-1)$ and $N=\left(0, \ldots, 0,-\frac{1}{2}, 1\right)$. Finally, let $K^{n}$ be the triangulation of $\left(S * M^{n-1}\right) \cup\left(N * M^{n-1}\right)$ with $n$-simplices $S * R * \sigma^{n-2}, \quad N * R * \sigma^{n-2}, \quad S N * \sigma^{n-2}$, and $S N * L * \eta^{n-3}$ for all $(n-3)-$ simplices $\eta^{n-3} \in \partial \sigma^{n-2}$. (This is a triangulation since $S N$ passes through the interior of $L * \sigma^{n-2}$.) Clearly, $K^{n}$ is strictly convex and has $n+3$ vertices. However, the strictly convex SL embedding $f: \partial K^{n} \rightarrow \mathbb{R}^{n}$ given by $f(N)=\left(0, \ldots, 0, \frac{1}{2}, 1\right)$, and $f$ fixes all other vertices, cannot be extended to an SL embedding of $K^{n}$ (since $f(S N)$ intersects the interior of $f\left(R * \sigma^{n-2}\right)$ ). See Fig. 2 for the threedimensional case; although $K^{3}$ is a triangulated octahedron, $K^{n}$ is not in general a triangulated higher-dimensional octahedron.

To prove minimality, suppose $N^{n} \subset \mathbb{R}^{n}$ is a strictly convex $n$-ball with $n+2$ boundary vertices, and let $f: \partial N^{n} \rightarrow \mathbb{R}^{n}$ be a strictly convex SL embedding. We will show that there is a unique projective homeomorphism $h: N^{n} \rightarrow$ convex hull $\left(f\left(\partial N^{n}\right)\right) \subset \mathbb{R}^{n}$ which agrees with $f$ on the vertices of $\partial N^{n}$. Let $F: N^{n} \rightarrow$ $\mathbb{R}^{n}$ be the unique SL map with $F(v)=h(v)$ for all vertices $v$ of $N^{n}$. It can be checked that $F$ is an embedding (see Lemma 4.4 of [6]), and, clearly, $F \mid \partial N^{n}=f$; hence $N^{n}$ with $n+2$ boundary vertices cannot satisfy the conditions of the theorem. If $N^{n}$ has $n+2$ vertices, with one in the interior, or if $N^{n}$ has $n+1$ 


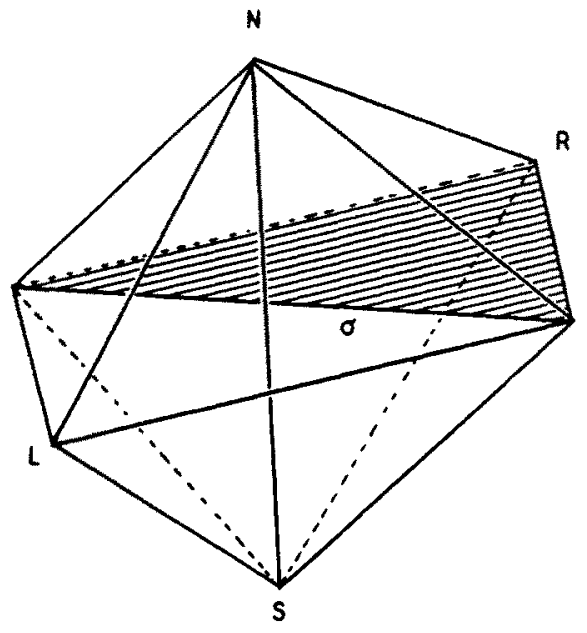

Fig. 2

vertices (all of which must lie on the boundary), $h$ can be taken to be affine linear, with similar conclusions. This proves that $K^{n}$ has the minimal number of vertices.

It remains to show that the projective homeomorphism $h: N^{n} \rightarrow$ convex hull $\left(f\left(\partial N^{n}\right)\right) \subset \mathbb{R}^{n}$ exists. Since $n+1$ points determine an affine linear map of $\mathbb{R}^{n}$, and since composing by such maps will not affect what we are trying to show, we may assume without loss of generality that $\partial N^{n}$ has vertices $a_{0}=$ $(0, \ldots, 0), \quad a_{1}=(1,0, \ldots, 0), \quad a_{2}=(0,1,0, \ldots, 0), \ldots, a_{n}=(0, \ldots, 0,1), \quad$ and $a_{n+1}=\left(c_{1}, c_{2}, \ldots, c_{n}\right)$, with $\sum_{i=1}^{n} c_{i}>1$, and $c_{i}>0$ for all $i$, and that $f\left(\partial N^{n}\right)$ has vertices $\quad a_{0}^{\prime}=(0, \ldots, 0), \quad a_{1}^{\prime}=(1,0, \ldots, 0), \quad a_{2}^{\prime}=(0,1,0, \ldots, 0), \ldots, a_{n}^{\prime}=$ $(0, \ldots, 0,1)$, and $a_{n+1}^{\prime}=\left(c_{1}^{\prime}, c_{2}^{\prime}, \ldots, c_{n}^{\prime}\right)$, with $\sum_{i=1}^{n} c_{i}^{\prime}>1$, and $c_{1}^{\prime}>0$ for all $i$, and also that $f\left(a_{i}\right)=a_{i}^{\prime}$.

Consider $\mathbb{R} P^{n}$ as having homogeneous coordinates $\left[x_{1}, x_{2}, \ldots, x_{n+1}\right]$ in the usual way. Let $I: \mathbb{R}^{n} \rightarrow \mathbb{R} P^{n}$ be the standard embedding $I\left(x_{1}, x_{2}, \ldots, x_{n}\right)=$ $\left[x_{1}, x_{2}, \ldots, x_{n}, 1\right]$; points in the $(n-1)$-plane at $\infty$ in $\mathbb{R} P^{n}$ are those with homogeneous coordinates $\left[x_{1}, x_{2}, \ldots, x_{n}, 0\right]$. Let $A_{i}, A_{i}^{\prime} \in \mathbb{R} P^{n}$ be the points given by $A_{i}=I\left(a_{i}\right)$ and $A_{i}^{\prime}=I\left(a_{i}^{\prime}\right)$ for all $0 \leq i \leq n+1$. Explicitly, $A_{0}=[0, \ldots, 0,1]$, $A_{1}=[1,0, \ldots, 0,1], \quad A_{2}=[0,1,0, \ldots, 0,1], \ldots, A_{n}=[0, \ldots, 0,1,1], \quad A_{n+1}=$ $\left[c_{1}, c_{2}, \ldots, c_{n}, 1\right], \quad$ and $\quad A_{0}^{\prime}=[0, \ldots, 0,1], \quad A_{1}^{\prime}=[1,0, \ldots, 0,1], \quad A_{2}^{\prime}=$ $[0,1,0, \ldots, 0,1], \ldots, A_{n}^{\prime}=[0, \ldots, 0,1,1], A_{n+1}^{\prime}=\left[c_{1}^{\prime}, c_{2}^{\prime}, \ldots, c_{n}^{\prime}, 1\right]$. For ease of notation, we identify $N^{n}$ with $I\left(N^{n}\right)$.

Because of the strict convexity of $\partial N^{n}$ and $f\left(\partial N^{n}\right)$ (considered as subsets of $\mathbb{R}^{n}$ ), it follows that no $n+1$ vertices of the $A_{i}$ or of the $A_{i}^{\prime}$ lie in an $(n-1)$-plane. A theorem of Projective Geometry (often referred to as the fundamental theorem of the subject) then implies that there is a projective homeomorphism $h: \mathbb{R} P^{n} \rightarrow$ $\mathbb{R} P^{n}$ such that $h\left(A_{i}\right)=A_{i}^{\prime}$ for all $i$. $N^{n}$ contains no points at $\infty$, and if we show that $n\left(N^{n}\right) \cap \infty=\varnothing$, then the restriction $h \mid N^{n}: N^{n} \rightarrow \mathbb{R}^{n}$ will be the desired map.

It can be checked that the $(n+1) \times(n+1)$ matrix $M$ representing $h: \mathbb{R} P^{n} \rightarrow \mathbb{R} P^{n}$ 
in homogeneous coordinates is given by

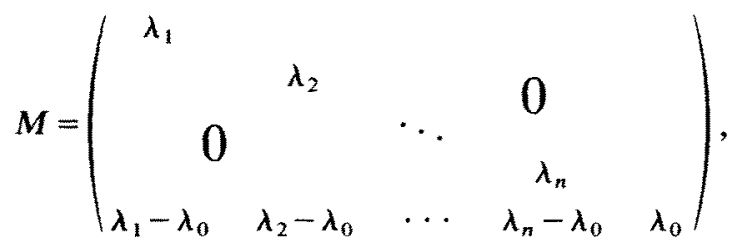

where $\lambda_{i}=c_{i}^{\prime} / c_{i}$ for $1 \leq i \leq n$, and $\lambda_{0}=\left(1-\sum_{i=1}^{n} c_{i}^{\prime}\right) /\left(1-\sum_{i=1}^{n} c_{i}\right)$. Note that the conditions on the $c_{i}$ and $c_{i}^{\prime}$ ensure that $\lambda_{j}>0$ for $0 \leq j \leq n$. A point in $\mathbb{R} P^{n}$ is at $\infty$ iff its $(n+1)$ st homogeneous coordinate is 0 . Hence, if $x=\left(x_{1}, x_{2}, \ldots, x_{n}\right) \in \mathbb{R}^{n}$, then $h(x)$ is at $\infty$ iff the vector $M X$ has $(n+1)$ st homogeneous coordinate 0 , where $X=\left[x_{1}, x_{2}, \ldots, x_{n}, 1\right]$. This condition yields the equation

$$
\left(\lambda_{1}-\lambda_{0}\right) x_{1}+\cdots+\left(\lambda_{n}-\lambda_{0}\right) x_{n}+\lambda_{0}=0
$$

i.e., $\left(x_{1}, \ldots, x_{n}\right) \cdot\left(\lambda_{1}-\lambda_{0}, \ldots, \lambda_{n}-\lambda_{0}\right)=-\lambda_{0}$, which is an $(n-1)$-plane in $\mathbb{R}^{n}$. It will thus suffice to show that this plane does not intersect $N^{n}$. However, it is easy to check that $a_{0} \cdot\left(\lambda_{1}-\lambda_{0}, \ldots, \lambda_{n}-\lambda_{0}\right)=0, a_{i} \cdot\left(\lambda_{1}-\lambda_{0}, \ldots, \lambda_{n}-\lambda_{0}\right)=\lambda_{i}-\lambda_{0}$ for $1 \leq i \leq n$, and $a_{n+1} \cdot\left(\lambda_{1}-\lambda_{0}, \ldots, \lambda_{n}-\lambda_{0}\right)=1-\lambda_{0}$, all of which are greater than $-\lambda_{0}$. Hence the vertices of $N^{n}$ all lie on the same side of the $(n-1)$-plane, and by convexity all of $N^{n}$ misses this plane. This concludes the proof that the map $h$ exists as desired.

\section{Example 3}

Let $T_{n} \subset \mathbb{R}^{n}$ be a snubbed regular $n$-simplex, which is the complex that is left after we slice off (with $(n-1)$-planes) neighborhoods of all the vertices of a regular $n$-simplex in $\mathbb{R}^{n}$; let $Q_{n} \subset \mathbb{R}^{n}$ be a snubbed regular $n$-cube, defined similarly. $T_{n}$ has $2 n+2(n-1)$-faces, of which $n+1$ are $(n-1)$-simplices, and $n+1$ are something else; $Q_{n}$ has $2^{n}+2 n(n-1)$-faces, of which $2^{n}$ are $(n-1)$ simplices, and $2 n$ are something else. We use $N_{n}$ to denote either $T_{n}$ or $Q_{n}$.

Consider $N_{n-1} \subset \mathbb{R}^{n-1}=\mathbb{R}^{n-1} \times\{0\} \subset \mathbb{R}^{n}$, with $N_{n-1}$ situated so that the origin $O$ of $\mathbb{R}^{n}$ is the center of gravity of $N_{n-1}$. Our example $K^{n}$ is a triangulation of $N_{n-1} \times[-1,1] \subset \mathbb{R}^{n}$. Choose $\varepsilon>0$ to be some very small number. For each $(n-2)$-face $\sigma$ of $N_{n-1}$, let $\sigma^{*}$ denote the center of gravity of $\sigma$. Define the hook for $\sigma$ to be the triangular 1 -sphere $H_{\sigma}=\left(\sigma^{*} \times[-\varepsilon, \varepsilon]\right) \cup\left(\sigma^{*} \times\{-\varepsilon, \varepsilon\}\right) * O$. Let $S_{n-2}$ be the boundary of an $(n-1)$-simplex which lies in the interior of $N_{n-1}$, and which contains $O$ in its interior. Note that $S_{n-2}$ links each of the hooks $H_{\sigma}$. Finally, let $K^{n}$ be a triangulation of $N_{n-1} \times[-1,1]$ which does not subdivide $S_{n-2}$ or any of the $H_{\sigma}$. Clearly, $K^{n}$ is convex, and can be chosen so as not to have any spanning simplices.

The $(n-2)$-faces $\sigma$ of $N_{n-1}$ are in two categories: those that are $(n-2)$ simplices, and those which are not. Let $\partial N_{n-1}^{(n-3)}$ denote the $(n-3)$-skeleton of $\partial N_{n-1}$. Because each set $\sigma \times[-1,1]$ is a flat $(n-1)$-ball in $\mathbb{R}^{n}$, standard PL 
techniques imply that $\partial K^{n}$ could have been triangulated finely enough, without subdividing any of the $\sigma^{*} \times[-\varepsilon, \varepsilon]$, so that there is an SL map $f: \partial K^{n} \rightarrow \mathbb{R}^{n}$ with the following properties:

(1) $f$ takes $\partial K^{n}$ homeomorphically onto itself.

(2) $f$ fixes $\partial N_{n-1}^{(n-3)} \times[-1,1]$ and $N_{n-1} \times\{-1,1\}$.

(3) If $\sigma$ is an $(n-2)$-face of $N_{n-1}$ which is an $(n-2)$-simplex, then $f\left(\sigma^{*} \times[-\varepsilon, \varepsilon]\right)=\sigma^{*} \times[p-\varepsilon, p+\varepsilon]$ for some $\varepsilon<p<1-\varepsilon$.

(4) If $\sigma$ is an $(n-2)$-face of $N_{n-1}$ which is not an $(n-2)$-simplex, then $f\left(\sigma^{*} \times[-\varepsilon, \varepsilon]\right)=\sigma^{*} \times[-p-\varepsilon,-p+\varepsilon]$ for $p$ as in $(3)$.

The map $f$ is a convex SL embedding of $\partial K^{n}$. The question is, can $f$ be extended to an SL embedding of $K^{n}$ ? The deciding issue is the following: can $f(O)$ be defined in a way such that $f\left(S_{n-2}\right)$ can be located so as to still link all the $f\left(H_{\sigma}\right)$ ? If $f(O)$ can be so chosen, then a fine enough triangulation of $K^{n}$ will allow $f$ to be extended to all of $K^{n}$; on the other hand, since an embedding preserves linking, if no such $f(O)$ existed, then $f$ could not be extended to an SL embedding $\boldsymbol{K}^{\boldsymbol{n}}$.

Whether $f(O)$ can be chosen as above depends on whether $N_{n-1}=T_{n-1}$ or $N_{n-1}=Q_{n-1}$. In either case, we first note that for $f(O)$ to be as required, it must have the $n$th coordinate greater than $p$ in absolute value (where $p$ is as in conditions (3) and (4) above). This is true because $f\left(S_{n-2}\right)$ must lie in an $(n-1)$-plane in $\mathbb{P}^{n}$, and if the $n$th coordinate of $f(O)$ is less than or equal to $p$ in absolute value, there is no $(n-1)$-plane which intersects all the $f\left(H_{\sigma}\right)$. Figure 3 shows the case for $n=3$, using $N_{2}=T_{2}$ (which is a hexagon).

In the case $N_{n-1}=Q_{n-1}$, it is in fact seen that the number of hooks, and their locations, do not allow $f(O)$ to be defined as required even with the $n$th coordinate greater than $p$ in absolute value. Thus $f$ cannot be extended to an SL embedding of $K^{n}$, yielding the example of Theorem 5(ii).

In the case $N_{n-1}=T_{n-1}$, it is seen that for appropriate choices of the number
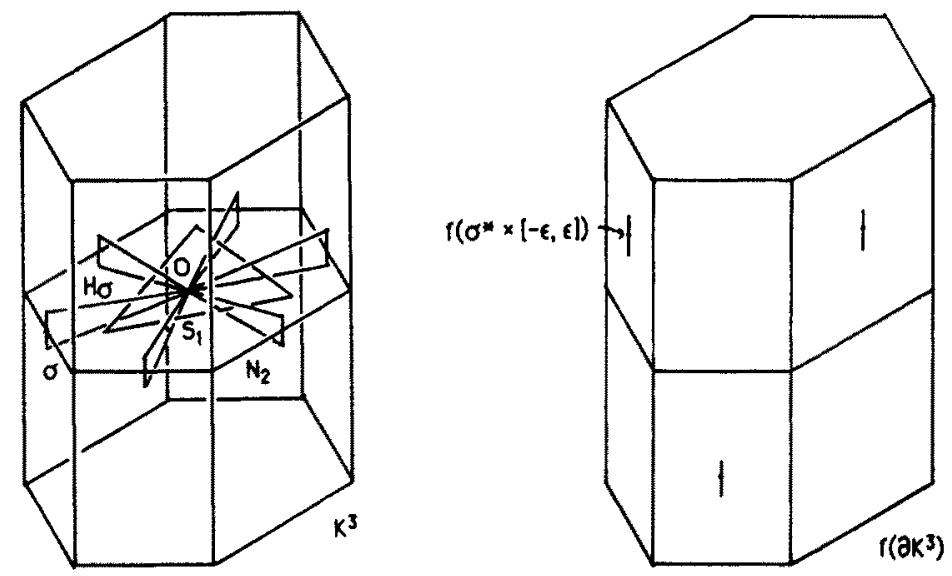

Fig. 3 
$p$, there are choices of $f(O)$ as required. For appropriate choices of $p$ (obtained by the Intermediate Value Theorem, by examining what happens for $p$ very close to $\varepsilon$, and $p$ very close to $1-\varepsilon$ ), it is seen that $f(O)$ may be chosen so that the following holds: $f\left(S_{n-2}\right)$ can be located so as to link all the hooks $f\left(H_{\sigma}\right)$, where $f\left(S_{n-2}\right)$ links $f\left(H_{\sigma}\right)$ at a vertex of $f\left(S_{n-2}\right)$ if $\sigma$ is an $(n-2)$-simplex, and $f\left(S_{n-2}\right)$ links $f\left(H_{\sigma}\right)$ at a barycenter of an $(n-2)$-face of $f\left(S_{n-2}\right)$ if $\sigma$ is not an $(n-$ 2 ) -simplex. By symmetry, there must be choices of $f(O)$ with the $n$th coordinate greater than $p$, as well as with the $n$th coordinate less than $-p$. Let $F_{1}, F_{2}: K^{n} \rightarrow \mathbb{R}^{n}$ be SL embeddings extending $f$ such that $F_{1}(O)$ has the $n$th coordinate greater than $p$, and $F_{2}(O)$ has the $n$th coordinate less than $p$. Note that $F_{1}$ and $F_{2}$ are actually maps $K^{n} \rightarrow K^{n}$. The map $F_{2} \circ F_{1}^{-1}: K^{n} \rightarrow K^{n}$ is an SL homeomorphism fixing $\partial K^{n}$ pointwise, i.e., $F_{2} \circ F_{1}^{-1} \in L\left(F_{1}\left(K^{n}\right)\right)$, in the notation of the introduction. We observe that $L\left(F_{1}\left(K^{n}\right)\right)$ is not connected, since there can be no path from $F_{2} \circ F_{1}^{-1}$ to the identity map $i d \in F_{1}\left(K^{n}\right)$. Note that $F_{2} \circ F_{1}^{-1}(O)<-p$, whereas $i d(O)>p$; the Intermediate Value Theorem then implies that, for any path from $F_{2} \circ F_{1}^{-1}$ to $i d$, at some point the image of $O$ must have the $n$th coordinate equal to 0 , an impossibility. Hence $L\left(F_{1}\left(K^{n}\right)\right)$ is the example of Theorem 2.

Question 2. What conditions on $K^{n}$ ensure that the analog of Bing and Starbird's theorem (Theorem 3 of the Introduction) holds? One guess is that for any $K^{n}$, the result would hold for the second barycentric subdivision of $K^{n}$.

It should be noted that if we consider SL maps of $\partial K^{n}$ which have nonconvex images, the situation is much more complicated, since it is complicated even in two dimensions; see [2] and [11].

\section{Acknowledgment}

The author would like to thank Les Reid and the referee for helpful suggestions.

\section{References}

1. R. H. Bing and and M. Starbird, Linear isotopies in $E^{2}$, Trans. Amer. Math. Soc. 237 (1978), $205-222$.

2. R. H. Bing and M. Starbird, Super triangulations, Pacific J. Math. 74 (1978), 307-325.

3. E. D. Bloch, Simplexwise linear near-embeddings of a 2. disk into $\mathbb{R}^{2}$, Trans. Amer. Math. Soc. $288(1985), 701-722$.

4. E. D. Bloch, Strictly convex simplexwise linear near-embeddings of a 2-disk, Trans. Amer. Math. Soc. 288 (1985), 723-737.

5. E. D. Bloch, Simplexwise linear near-embeddings of a 2-disk ino $\mathbb{R}^{2}$, I1, Topology Appl. 25 (1987), 93-101.

6. E. D. Bloch, R. Connelly, and D. W. Henderson, The space of simplexwise linear homeomorphisms of a convex 2-disk, Topology 23 (1984), 161-175.

7. R. Connelly and D. W. Henderson, A convex 3-complex not simplicially isomorphic to a strictly convex complex, Math. Proc. Cambridge Philos. Soc. 88 (1980), 299-306. 
8. R. Connelly, D. W. Henderson, C.-W. Ho, and M. Starbird, On the problems related to linear homeomorphisms, embeddings, and isotopies, in Continua, Decompositions, Manifolds, University of Texas Press, Austin, TX, 1983.

9. B. Grünbaum, Convex Polytopes, Wiley, New York, 1967.

10. C.-W. Ho, On the space of the linear homeomorphisms of a polyhedral $n$-cell with two interior vertices, Math. Ann. 243 (1979), 227-236.

11. C. W. Ho, On the extendability of a linear embedding of the boundary of a triangulated $n$-cell to an embedding of the $n$-cell, Amer. J. Math. 103 (1981), 1185-1193.

12. P. Mani, Spheres with few vertices, J. Combin. Theory Ser. A 13 (1972), 346-352.

13. M. Starbird, A complex which cannot be pushed around in $E^{3}$, Proc. Amer. Math. Soc. 63 (1977), 363-367.

Received September 15, 1986, and in revised form April 15, 1988. 